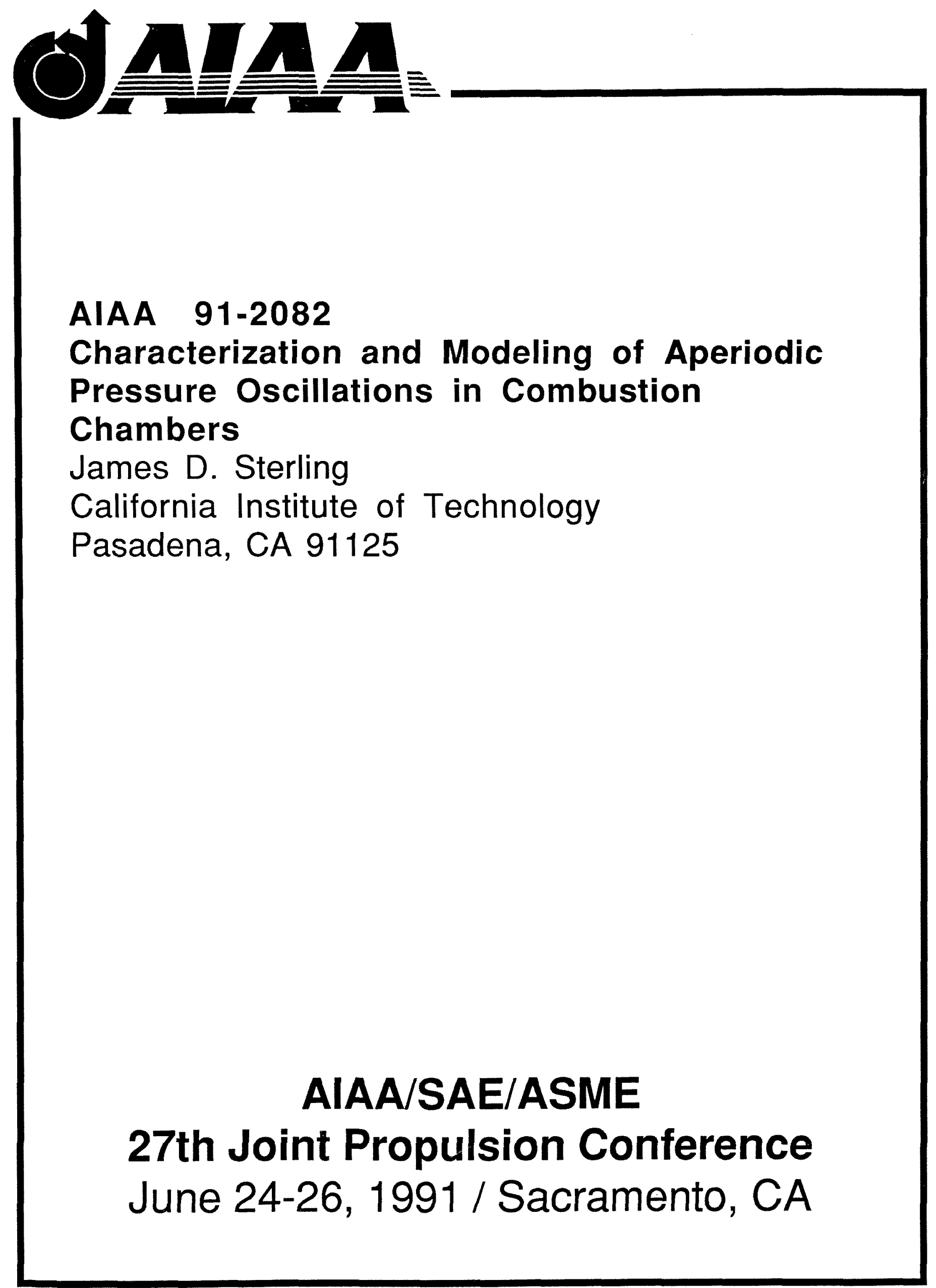




\title{
CHARACTERIZATION AND MODELING OF APERIODIC PRESSURE OSCILLATIONS IN COMBUSTION CHAMBERS
}

\author{
James D. Sterling* \\ California Institute of Technology \\ Jet Propulsion Center 301-46 \\ Pasadena, California 91125
}

\begin{abstract}
$\underline{\text { Abstract }}$
Classification of the long-term dynamical behavior of pressure oscillations in a laboratory combustion chamber has been performed using methods of modern dynamical systems theory. The method involves the construction of a phase-space representation from a single pressure record or time series using the time-delay embedding method. The pointwise correlation dimension of the resulting attractor in phase-space provides a lower bound on the number of modes that participate in the oscillations. The results show that the oscillations are quasiperiodic with a dimension near two over an order of magnitude of amplitudes. Quasiperiodicity is a result of the incommensurate frequencies of the system acoustic modes. A model for the dynamics is constructed by converting the governing equations to a kicked-oscillator model. When compared with the experimental data, the model results have similar pressure and velocity spectra and the attractor dimension verifies that quasiperiodic oscillations are present.
\end{abstract}

\section{Introduction}

Spectrum analysis of pressure signals obtained during combustion instability generally reveals large peaks at natural acoustic modes of the system. This observation suggests that while a highly nonlinear, infinite-dimensional system of turbulent combustion exists, low-dimensional behavior may be dominant at the large amplitudes, or large scales, of the oscillatory motion. Developments in dynamical systems theory during the last decade have resulted in the advent of dimension-finding algorithms that may be used to obtain a lower bound on the number of modes that participate in the dynamics of pressure oscillations.

These system identification techniques are based on the idea of characterizing a dynamical system by the topology of an attractor that appears in a phase-space representation of the system dynamics. The methods were developed for analysis of nonlinear differential equations but the extension to experimental data is performed by placing or embedding an experimental time series in a phase space representation. The original work on this subject by Packard et al. (1979) has been followed by many refinements of the technique which has been applied to time series from many fields of study. Recently, Gershenfeld (1987) and Theiler (1990) have reviewed the methods of dimension determination of time series.

The presence of a low-dimensional attractor associated with experimental data provides justification that low-dimensional deterministic modeling may be sufficient to capture the dominant behavior of the system. In the case of combustion instability, Culick $(1976,1990)$ has used a version of Galerkin's method to convert the governing partial differential equations into a set of coupled ordinary differential equations. This is accomplished by using an expansion of the oscillatory fields in terms of orthogonal acoustic modes of the combustion chamber. The resulting equations provide a framework for low-dimensional models to be constructed while retaining many physically relevant phenomena. 


\section{Characterization}

Time-Delay Embedding Method

The first step in determining the dimension of an experimental signal, $p(t)$, is to place or embed the signal into an n-dimensional phase space by constructing the following trajectory, $\mathbf{P}(t)$,

$$
P(t)=\{p(t), p(t+\tau), p(t+2 \tau), \ldots . p(t+n \tau)\}
$$

where $\mathrm{n}$ is the embedding dimension and $\tau$ is an arbitrary time delay. This is known as the time-delay embedding method and has become a commonly used tool for studies of nonlinear dynamical systems. If $p(t)$ is a digitized time series then the phase-space representation is a set of points that constitute an attractor. With a phase-space representation of the signal, the dimension of the attractor may be determined.

\section{Attractor Dimension Determination}

The dimension estimate is obtained by considering an $n$-dimensional ball of radius $r$ surrounding a point on the attractor. The number of points within the ball is obtained as a function of the size of the ball. The number of points in the ball is expected to scale with the ball radius raised to the power $d$, where $d$ is the dimension of the attractor.

$$
N(r)=A r^{d}
$$

Therefore, $\log (\mathrm{N}(\mathrm{r}))$ is plotted versus $\log (\mathrm{r})$ and the slope represents the dimension of the attractor. Thus, a plot of the slope of this curve versus $\log (\mathrm{r})$ represents the pointwise dimension as a function of the ball radius for the point on the attractor used as the reference. Averaging for many points may be used to obtain statistical characteristics.

Since experimental data contains noise, at the smallest scales the attractor is infinitedimensional. In other words, for small ball radii about the reference point, the other points will randomly fill the embedding space. For ball radii as large as the size of the attractor, the dimension will be equal to zero. Thus, the dimension is expressed as a function of the magnification at which the attractor is observed. Over intermediate scales if the dimension reaches a plateau of constant value, $d$, then characterization by this single dimension may be appropriate. However, as discussed by Sauer et al. (1991) if the embedding dimension is less than $2 d+1$, it should be increased and the dimension recomputed.

This procedure does not always converge as shown by the example of a stochastic signal (i.e. an infinite-dimensional system). A random number generator was used to generate a 10000 point time series which was embedded into 5-dimensional and 9-dimensional phase space trajectories. The system appears to be zero-dimensional at the largest scales, but as seen in Figure 1, the dimension quickly approaches the embedding dimension as the scale is decreased. However, while 10000 points are satisfactory for the stochastic signal embedded in 5-d to provide the known asymptotic value of five, in the 9-d embedding more points are needed to resolve the dimension to be equal to the embedding dimension. In general, at least $10^{\mathrm{n}}$ points, where $\mathrm{n}$ is the embedding dimension, are needed to adequately resolve the dimension of an attractor. This puts severe computational constraints on the determination of dimension for systems with more than a few degrees of freedom.

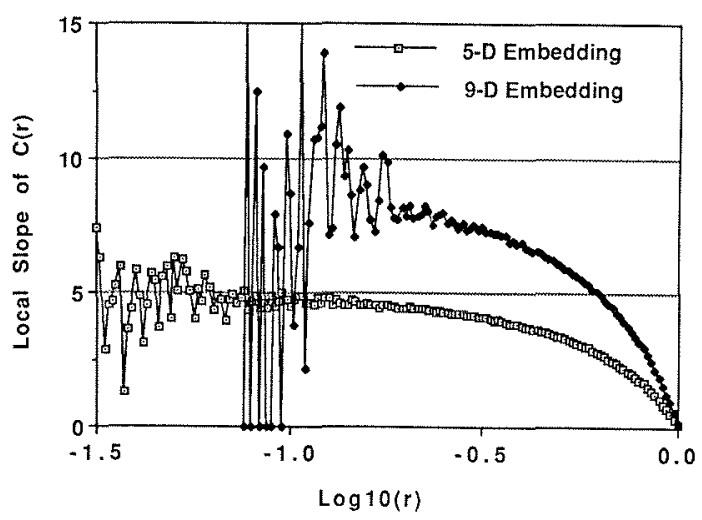

Figure 1: Dimension Determination Applied to Noise

The above results are presented to demonstrate the way in which one may determine if low-dimensional behavior is not present in a time series. Note that results 
similar to those in Figure 1 could arise from any system in which the effective number of degrees of freedom is greater than or equal to the embedding dimension. Numerous results of the method applied to low-dimensional attractors associated with known mathematical constructs have been presented by Gershenfeld (1987) and Theiler (1990).

\section{$\underline{\text { Results }}$}

Pressure signals taken from a combustor facility at the Jet Propulsion Center of Caltech have been characterized using the above methods. A rearward-facing step was used as the flameholder. Instrumentation included pressure transducers at many locations, a hotwire anemometer located upstream of the combustion region, and a photomultiplier tube to measure chemiluminescence from the flame. Details about the experiment can be found in Sterling (1987) and Sterling and Zukoski (1991).

The particular operating conditions considered here made use of a mixture of $30 \%$ hydrogen and $70 \%$ methane with air used as the oxidizer at a lean equivalence ratio of 0.7 . The mean velocity in the 4.8 square centimeter flow area above the step was 30 meters per second. These conditions resulted in large pressure oscillations and the shedding of large vortex structures from the step that were observed using a high speed camera and the shadowgraph technique.

The spectrum obtained from the pressure signal measured above the flameholder is presented in Figure 2. The peaks at $460 \mathrm{~Hz}$ and at $530 \mathrm{~Hz}$ correspond to acoustic modes of the system that were computed as eigenvalues of a one-dimensional acoustic model of the system. Other modes near 190, 233, and 385 were also computed and mode shapes were plotted by Sterling (1987). As the $530 \mathrm{~Hz}$ peaks shifts slightly in different experiments, the $265 \mathrm{~Hz}$ and $424 \mathrm{~Hz}$ peaks are observed to shift as well, leading to the conclusion that these peaks correspond to the $1 / 2$ and $4 / 5$ subharmonics of the $530 \mathrm{~Hz}$ mode.

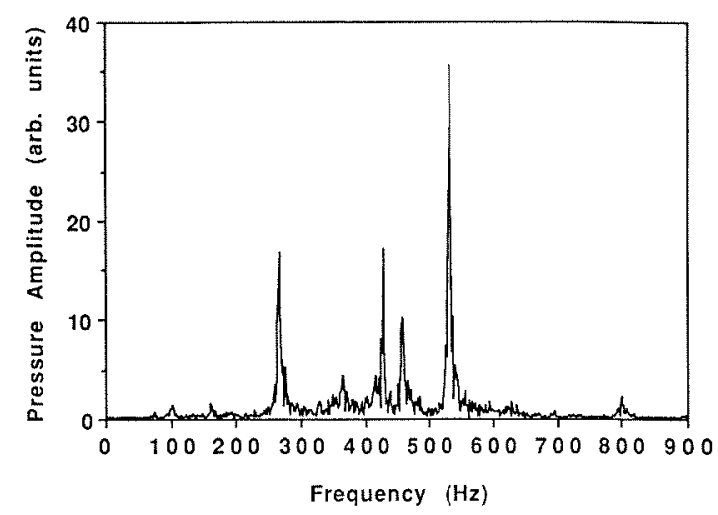

Figure 2: Spectrum of Pressure Signal During Combustion Instability

The pointwise dimension calculations for the pressure signal are presented as a function of the radius of the $n$-dimensional sphere in Figures 3 and 4 . The embedding dimension was equal to nine and 8150 points taken at 80 microsecond intervals were used to characterize the attractor.

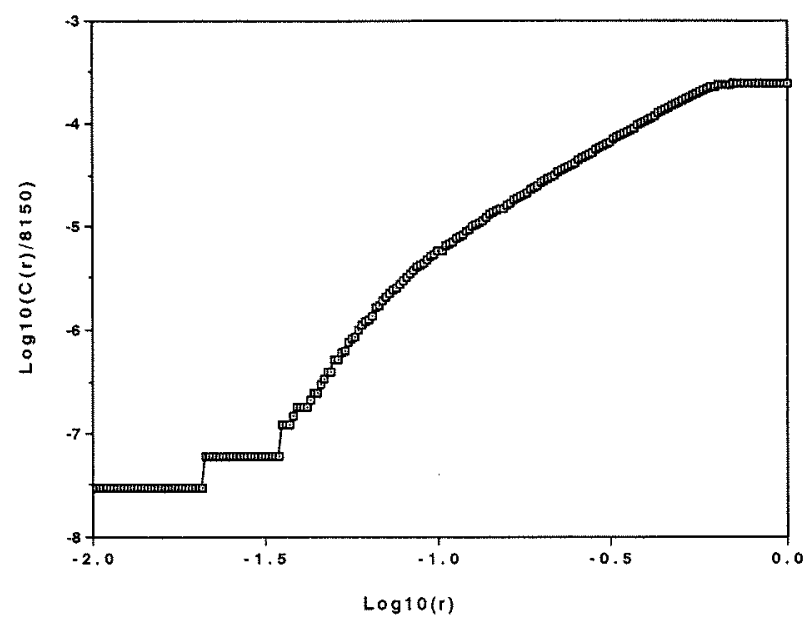

Figure 3: Number of Points Inside 9-D Ball Plotted Versus Radius 


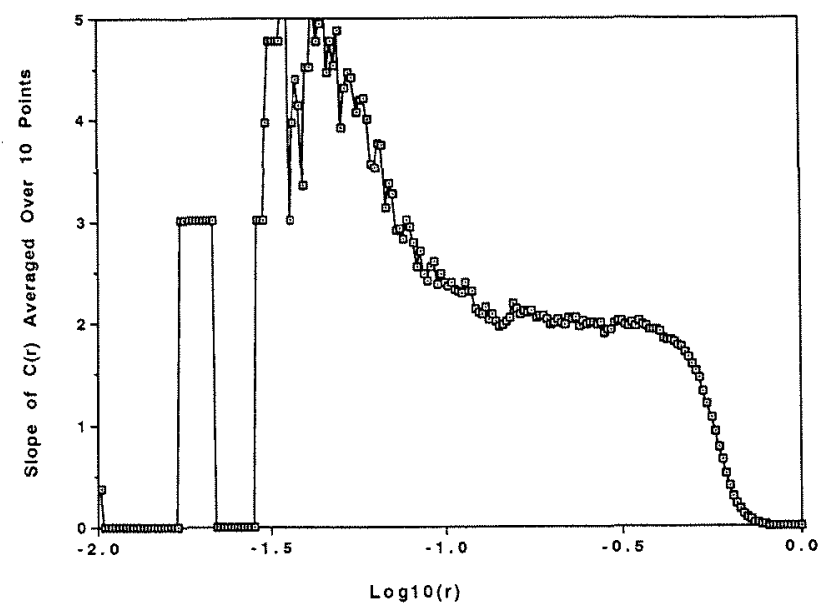

Figure 4: Pointwise Dimension of Pressure Signal with Spectrum in Figure 2

The slope of the curve in Figure 3 averaged over 10 points is plotted in Figure 4. The large plateau in Figure 4 near the value of two indicates that for oscillations over approximately one order of magnitude, the attractor is two-dimensional. This is consistent with the conclusion drawn from the spectra that two acoustic modes are participating in the oscillations. Thus, quasi-periodic behavior of two modes is present and nonlinearities are responsible for the appearance of the subharmonics in the spectra.

The quasiperiodicity arises because the ratio of the frequencies associated with the two eigenmodes is irrational or the frequencies are incommensurate. Systems in which the ratio of frequencies is rational or commensurate result in periodic oscillations that constitute a onedimensional attractor in phase space. This includes frequencies that are integer multiples of one another as is the case for simple longitudinal modes of chambers with constant area and sound speed.

In systems that have incommensurate frequencies, if the nonlinearities are strong enough the oscillations may become modelocked or entrained into a periodic orbit. The parameter ranges over which this entrainment occurs are referred to as Arnold tongues in parameter space. As shown in Figure 4, a onedimensional attractor corresponding to entrainment is not present in the laboratory combustor for the particular operating conditions but a two-dimensional quasiperiodic attractor is present.

Quasiperiodic behavior occurs without the subharmonic content when a stoichiometric mixture of methane and air is supplied at a flow speed of 18.3 meters per second. As shown in the pressure spectrum of Figure 5 , the $190 \mathrm{~Hz}$ and $460 \mathrm{~Hz}$ modes are both present. The other spectra in Figure 5 reveal that both the velocity at the flameholder and the radiation intensity 11.9 centimeters downstream of the flameholder are essentially periodic with the $460 \mathrm{~Hz}$ signal conspicuously absent. The reason for this apparent periodicity will be presented in the modeling of this case in the following section.
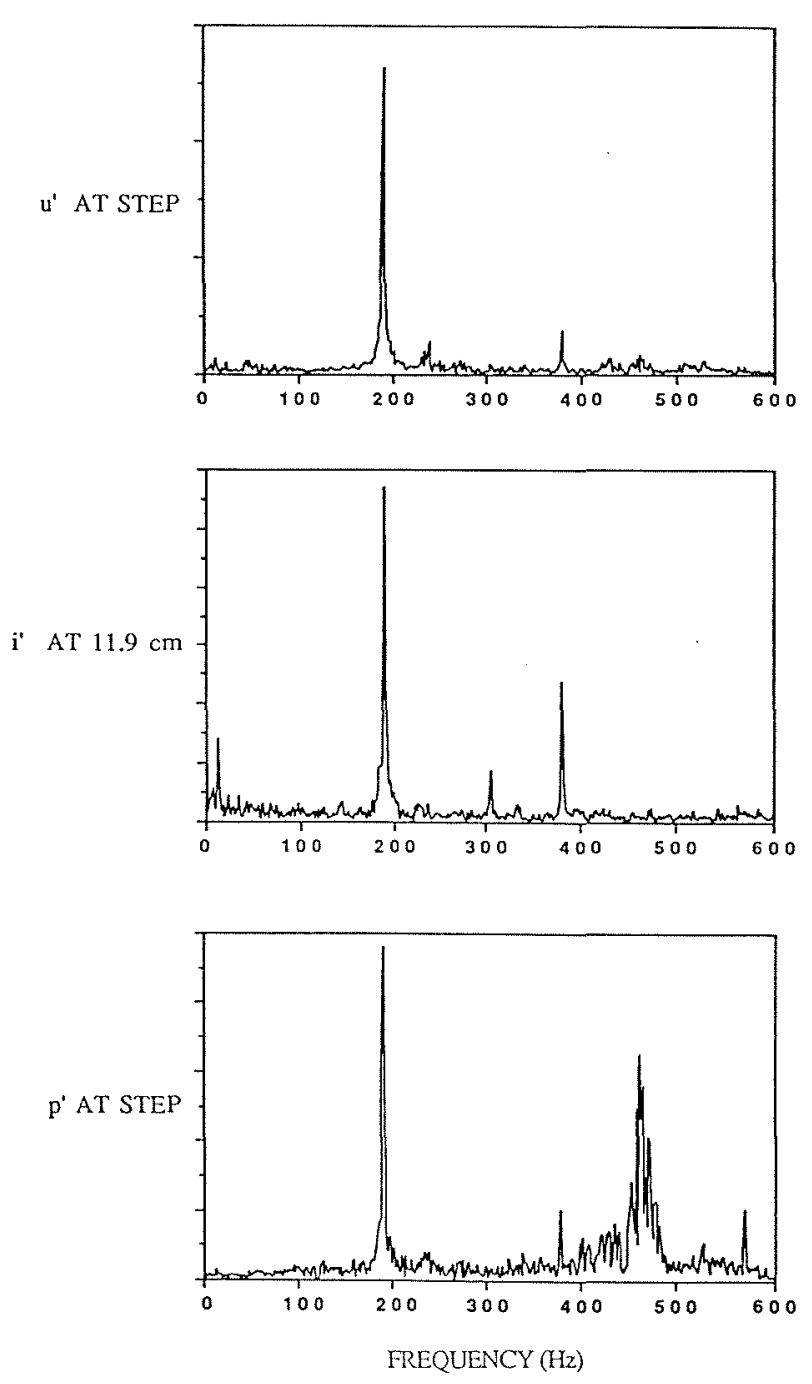

Figure 5: Spectra of Velocity, Radiation Intensity, and Pressure Under Different 
The dimension results presented above and previous dimension measurements of laboratory combustor pressure oscillations by Keanini et al. (1989) indicate that low-dimensional behavior dominates the large scale oscillatory motion.

\section{Modeling Aperiodic Behavior}

Since the pressure data reveals that lowdimensional behavior arises out of an infinitedimensional system, modeling is expected to result in the reduction of the governing equations to the measured dimensionality. A methodology using an expansion of the oscillatory pressure in system acoustic modes with time-varying amplitudes has been developed by Culick $(1976,1990)$. The method results in a set of ordinary differential equations for the modal amplitudes. Thus, a low-dimensional model that retains many physical processes can be constructed. If energy addition and damping mechanisms are adequately modeled, the phase-space trajectories, which represent the solution of the equations, will collapse onto attractors of low dimension. Comparison of the phase-space attractor dimensions of the models with those of the data can be performed to assess the validity of the model.

\section{Formulation}

A spectral representation of unsteady velocity and pressure components in terms of the normal acoustic modes of the system is derived as follows. Details will not be reproduced here but a framework will be provided for reference. By neglecting body forces but including heat sources, the inviscid and non-conducting conservation equations may be written in the following form,

$$
\begin{gathered}
\frac{\partial \rho}{\partial t}+\nabla \cdot(\mathbf{u} \rho)=0 \\
\rho \frac{\partial \mathbf{u}}{\partial t}+\rho \mathbf{u} \cdot \nabla \mathbf{u}=-\nabla p \\
\frac{\partial p}{\partial t}+\gamma p \nabla \cdot \mathbf{u}+\mathbf{u} \cdot \nabla p=(\gamma-1) q
\end{gathered}
$$

pressure, and velocity and $q$ is the rate of heat addition. By expanding these quantities into mean and fluctuating components, a wave equation for the pressure fluctuations can be obtained;

$$
\frac{\partial^{2} p^{\prime}}{\partial t^{2}}-a^{2} \nabla^{2} p^{\prime}=(\gamma-1) \frac{\partial q^{\prime}}{\partial t}
$$

A form of the method of weighted residuals can be used to solve this equation for given boundary conditions,

$$
\hat{n} \cdot \nabla p^{\prime}=-f
$$

where $\mathrm{n}$ is the outward normal vector on the boundaries of the chamber. A solution for the oscillatory pressure is expanded in the orthogonal acoustic modes of the system, $\psi_{n}$, that are defined by the eigenvalue problem,

$$
\begin{aligned}
& \nabla^{2} \psi_{n}+k_{n}^{2} \psi_{n}=0 \\
& \hat{n} \cdot \nabla \psi_{n}=0
\end{aligned}
$$

where $k_{n}=\omega_{n} / a$ is the wavenumber for the $n^{\text {th }}$ mode having frequency $\omega_{n}$. The expansion is given by time-varying amplitudes of the normal modes,

$$
p^{\prime}=\bar{p} \sum \eta_{n}(t) \psi_{n}(\mathbf{r})
$$

This expression is substituted into Equation 6 and multiplied by Equation 8. Equation 6 is then multiplied by $\psi_{n}$ and the two equations are then subtracted and integrated over the chamber volume. By using the orthogonality of the acoustic modes, Green's theorem, and by considering ideal boundary conditions $(f=0)$, a set of ordinary differential equations for the modal coefficients is produced such that

$$
\frac{d^{2} \eta_{n}}{d t^{2}}+\omega_{n}^{2} \eta_{n}=\frac{\gamma-1}{p E_{n}^{2}} \int \psi_{n} \frac{\partial q^{\prime}}{\partial t} d V
$$

where $r, p$, and the vector $\mathbf{u}$ are the density, 
where

$$
E_{n}^{2}=\int \psi_{n}^{2} d V
$$

Thus, the pressure variations in a combustion chamber are expressed in terms of a set of coupled oscillators that are driven by the heat release integral on the right side of Equation 11. An autonomous system can be obtained by including a nonlinear model for the heat release integral.

The right side of equation 11 is presented as a single term that represents driving of the acoustic oscillations by oscillatory heat release. The motivation for including only this driving term is based on previous work (Sterling and Zukoski, 1991) in which limiting amplitudes of pressure oscillations were observed to be governed by Rayleigh's mechanism of energy transfer. In this study, energy transfer to the acoustics of a laboratory combustor by "in-phase" heat release and pressure oscillations was observed to be balanced by damping due to "out-of-phase" heat release and pressure oscillations. Thus, limiting amplitudes were observed to occur as a result of damping associated with Rayleigh's mechanism.

The amplitudes of energy addition and subtraction by this mechanism were observed to be quite large compared to estimated contributions from other mechanisms of damping such as viscous boundary layer losses and non-ideal acoustic boundary conditions. For this reason, terms other than Rayleigh's mechanism of energy transfer have been neglected in equation 11.

\section{Heat Release Modeling}

A particular form of the heat release response to pressure and velocity fluctuations may be inserted into the right side of equation 11. If the heat release is expressed as a linear function of the pressure amplitude and its derivative, i.e. velocity, then stability can be investigated. If a nonlinear response is modeled then dynamical systems theory may be used to investigate the types of attractors that result.
The heat release response is therefore the most critical component of a model and is very system dependent. The physical mechanisms responsible for coupling the acoustics to the heat release for liquid propellant, solid propellant, and gas-fired systems are numerous and may vary with the geometry or operating conditioins of a given system. Thus, while linear analysis allows some generality for heat-release modeling (e.g. $n-t$ models or other models that yield a constant growth rate and frequency shift for each mode), generality can not be expected for nonlinear heat-release models. Therefore, only modeling of the vortex shedding feedback mechanism in premixed combustion systems will be addressed here.

\section{Vortex Shedding-Kicked Oscillator Model}

As previously mentioned, large pressure oscillations are observed to coincide with the shedding of large vortex structures from the flameholder. The model for the heat release coupling to the acoustics in this case is formulated as follows.

The amplitude of the oscillatory velocity at the flameholder is expressed as the sum of the velocities of each of the acoustic modes. This requires the gradient of the mode shape, $\mathrm{Y}$, and the amplitude, $\mathrm{h}$, of each mode. The oscillatory velocity at the flameholder results in the shedding of large vortex structures as the velocity increases from a minimum. This effect is modeled as the shedding of a vortex at the time of the minimum in the velocity at the flameholder.

The convection of the vortex structure and the mixing of fresh reactants with hot products of combustion results in a delay between the shedding of the structure and the chemical reaction of the reactants. This delay is modeled to be proportional to the magnitude of the oscillatory velocity at the time of shedding. The proportionality constant is the first adjustable model parameter and it is made dimensionless by normalizing with the period of first oscillator and the mean flow velocity.

$$
\tau=C_{1} \mid u_{\min }(\text { step }) \mid /\left(\omega 1 u_{\text {mean }}\right)
$$


A delay that increases with the velocity magnitude may be a result of a variety of mechanisms. Large velocity fluctuations yield large vortex structures. One postulated mechanism involves quenching of the flame by cool chamber walls that occurs when a large vortex structure grows to fill the entire chamber height. This effect delays the combustion of the reactants more than when small vortex structures (which are entirely surrounded by hot products of combustion) are shed. Another possible mechanism involves the ignition delay due to the kinetics of a volume of fresh reactants mixed with hot products of combustion. The larger vortex structures simply provide more cold products to the volume resulting in longer kinetic ignition delays.

The discussion above implicitly assumes that there is a single average delay from shedding to reaction of all the reactants associated with a single vortex structure. If the reaction is modeled to occur at one location at one time, then equation 11 becomes a kicked oscillator that is in free oscillation until energy is added discontinuously when a vortex structure reacts. Kicked oscillator models are prototypes for nonlinear oscillations because they serve to reduce differential equations to difference equations that give Poincare' maps of differential systems.

The model used here assumes that the phase of the oscillation remains fixed across the kick, and the energy added to each mode is proportional to the magnitude of the velocity at the time the vortex is shed multiplied by the instantaneous amplitude of the pressure associated with each mode at the reaction location.

$$
\Delta E n=C_{n} \eta_{n} \Psi_{n} \mid u_{\min }(\text { step }) \mid / u_{\text {mean }}
$$

Note that the amplitude of the kick for a particular mode depends upon all of the mode shapes evaluated at the flameholder (for velocity) and the particular mode shape at the reaction location. Also, for each mode a new proportionality constant is introduced.
In summary, given some initial conditions for a fixed number of modes, the free oscillations are numerically computed until a minimum in velocity is reached. At that time the magnitude of the velocity evaluated at the flameholder is saved to determine the amplitude and delay of the kick to be applied to each oscillator. The discontinuous kicks of the oscillators are then applied and the longterm behavior is observed. Spectral analysis and dimension-determination techniques are used to compare the model with the experimental results.

\section{$\underline{\text { Results }}$}

The described program has been applied to model the laboratory combustor conditions that yield the spectra in Figure 5 . The gradient of the mode shapes of the $190 \mathrm{~Hz}$ and $460 \mathrm{~Hz}$ modes evaluated at the flameholder and the mode shapes evaluated at the reaction location that is approximately $12 \mathrm{~cm}$ downstream of the flameholder were used as inputs to the program. Also, the proportionality constant relating the delay to the velocity was set to give a delay of one cycle of the $190 \mathrm{~Hz}$ oscillation for a unit velocity amplitude. The proportionality constants relating the kick amplitude to the amplitude of each of the modes were set to small equal values.

Spectra of the modeled velocity and pressure oscillations at the flameholder are shown in Figure 6. As with the experimental spectra, the velocity is essentially periodic at $190 \mathrm{~Hz}$ whereas two large peaks corresponding to the two modal frequencies appear in the pressure spectrum.

The reason for this difference is that the velocity at the flameholder associated with the $460 \mathrm{~Hz}$ mode is very small because the flameholder is near a node in the velocity mode shape. Thus, the velocity fluctuations consist of only the $190 \mathrm{~Hz}$ mode which does not have a node in the velocity mode shape near the flameholder. However, the heat is released in a location that is not near a pressure node for either mode so that energy is added to both modes during a kick. 

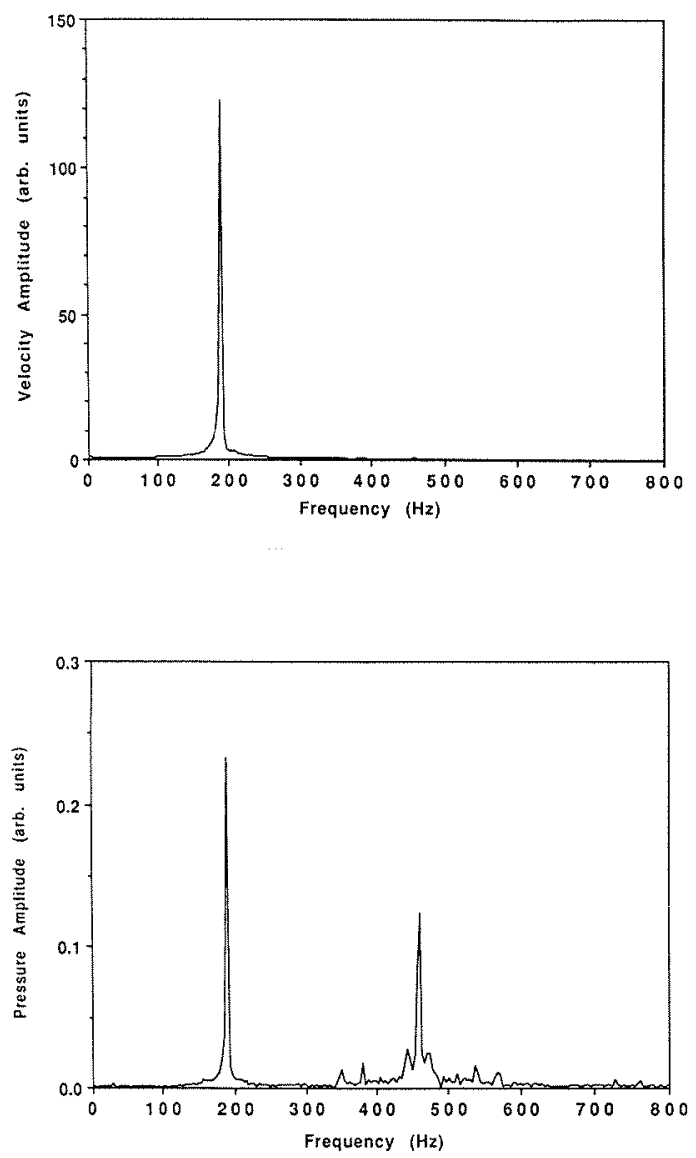

Figure 6: Spectra of Model Velocity and Pressure at Flameholder

When the time-delay embedding method and phase-space attractor dimension determination algorithms are applied, the results shown in Figure 7 are obtained. There is no single plateau that shows definite quasiperiodic behavior. However, the value does appear to be low-dimensional near the value of two. Thus, two modes driven by a delayed kick provide an infinite dimensional system that is observed to collapse down onto an attractor similar to the quasiperiodic attractor seen in the data.

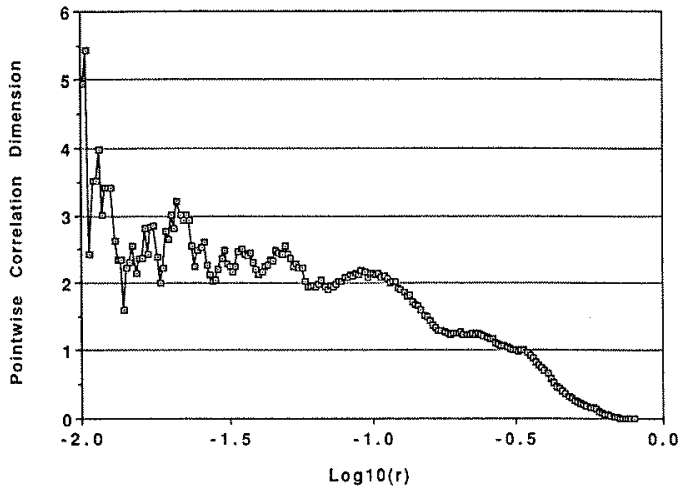

Figure 7: Pointwise Dimension of Model Pressure Signal

\section{Conclusions}

Time-series analysis techniques have been used to characterize pressure oscillations that occur during combustion instability of a laboratory combustor. The signal is first embedded into a phase-space representation using the time-delay embedding method. The dimension of the resulting phase-space attractor places a lower bound on the number of modes that participate in the oscillations.

Under a particular set of operating conditions, four large peaks in the pressure spectrum are observed; two corresponding to longitudinal acoustic modes of the system and two corresponding to subharmonics of the higher frequency mode. The dimension of the attractor in phase-space is verified to be nearly two over an order of magnitude of scales. Thus, quasiperiodic behavior involving two modes is observed.

The collapse of the large-scale behavior (governed by infinite-dimensional partial differential equations for the turbulent reacting flow) onto the low-dimensional attractor is modeled to occur as follows. First, a version of Galerkin's method is used to reduce the PDEs to a set of coupled ODEs for the amplitudes of the acoustic modes of the system. These equations contain a driving term due to the oscillatory heat release. The heat release associated with the shedding of large vortex structures from the 
flameholder is modeled to occur as the product of two delta functions; one in space and one in time. Thus, a nonlinear kicked-oscillator model is constructed that provides limitedamplitude oscillations for particular parameter values.

Comparison of power spectra and phasespace attractor dimension curves provides criteria for assessment of the validity of the model. A second set of operating conditions that generates quasiperiodic oscillations without the subharmonic content is modeled. The experimental pressure and velocity spectra are very similar to the spectra generated by the computational model. The pressure spectra show two large peaks at frequencies corresponding to longitudinal modes of the system. The velocity spectra consist of a single large peak corresponding the the vortex shedding frequency that is associated with the first acoustic mode of the system. The dimension of the model pressure signal attractor is measured to be close to two over an order of magnitude of scales.

In conclusion, the coupling of the acoustic oscillations with the heat release via the vortex shedding mechanism results in the linear self-excitation of only one mode because the higher frequency mode has a velocity node at the flameholder location. However, the nonlinear nature of the kicked-oscillator model allows energy to be fed into the higher frequency mode, resulting in quasiperiodic oscillations (i.e. an attractor dimension of two) of limited amplitude. This result has important consequences for investigations of combustion instability because it means that no two cycles of oscillation are identical and care must be taken to characterize the differences that occur in the combustion process for various cycles.

\section{References:}

Culick, F.E.C. (1976a,b) "Nonlinear Behavior of Acoustic Waves in Combustion Chambers." Parts I and II. Astronautica Acta 3.

Culick, F.E.C. (1990) "Some Recent Results for Nonlinear Acoustics in Combustion Chambers," AIAA-90-3927, (Conf. Proc. 13th Aeroacoustics Conference).

Gershenfeld, N. (1988) "An Experimentalist's Introduction to the Observation of Dynamical Systems," Chapter in Directions in Chaos, Vol 2., Hao Bai-lin (Ed.), World Scientific, Singapore.

Keanini, R., Yu, D., Daily, J. (1989) "Evidence of a Strange Attractor in Ramjet Combustion," AIAA-89-0624 (Conf. Proc. 27th Aerospace Sciences Meeting).

Packard, N.H., Crutchfield, J.P., Farmer, J.D. and Shaw, R.S. (1980) "Geometry from a Time Series," Physical Review Letters, Vol 45, No. 9, p. 712.

Sauer, T., Yorke, J.A., Casdagli, M. (1991) "Embedology," Santa Fe Institute Publication 91-01-008.

Sterling, J. D. (1987) Longitudinal Mode Combustion Instabilities in Air Breathing Engines. PhD thesis, California Institute of Technology, Pasadena, California.

Sterling, J.D. and Zukoski, E.E. (1991) "Nonlinear Dynamics of Laboratory Combustor Pressure Oscillations," Combustion Science and Technology, Vol. 77, No. 4-6, p. 225.

Theiler, J. (1990) "Estimating the Fractal Dimension of Chaotic Time Series," The Lincoln Laboratory Journal, Vol. 3, No. 1, pp. 63-86. 